\title{
Verification of generalized Kramers-Krönig relations and sum rules on experimental data of third harmonic generation susceptibility on polymers
}

\author{
Valerio Lucarini \\ Kai-Erik Peiponen \\ Department of Physics University of Joensuu \\ FIN-80101 Joensuu Finland
}

November 21, 2018

\begin{abstract}
We present the first analysis of harmonic generation data where the full potential of the generalized nonlinear Kramers-Krönig (KK) relations and sum rules is exploited. We consider two published sets of wide spectral range experimental data of third harmonic generation susceptibility on different polymers, the polysilane (frequency
\end{abstract}


range: $0.4-2.4 \mathrm{eV}$ ) and the polythiophene (frequency range: $0.5-2.0$ $e V)$. We show that, without extending the data outside their range with the assumption of an a-priori asymptotic behavior, independent truncated dispersion relations connect the real and imaginary part of the moments of the third harmonic generation susceptibility $\omega^{2 \alpha} \chi^{(3)}(3 \omega ; \omega, \omega, \omega)$, with $\alpha$ ranging from 0 to 3 , in agreement with the theory, while there is no convergence if we choose $\alpha=4$. We repeat the same analysis for $\omega^{2 \alpha}\left[\chi^{(3)}(3 \omega ; \omega, \omega, \omega)\right]^{2}$ and show that a larger number of independent $\mathrm{K}-\mathrm{K}$ relations connect the real and the imaginary part of the function under examination. We also compute the sum rules for the suitable moments of the real and imaginary parts, and observe that only considering higher powers of the susceptibility the correct vanishing sum rules are more precisely obeyed. All our results are in fundamental agreement to recent theoretical findings. Sum rules providing explicit information about structural properties of the material seem to require wider spectral range. These constraints are expected to hold for any material and provide fundamental tests of self-consistency that any experimental or model generated data have to obey; similar tests of coherence can be performed for other nonlinear optical processes, e.g. pump-and-probe. Verification of K-K relations and sum rules constitute unavoidable benchmarks for any investigation that addresses the nonlinear response of matter to radiation on a wide spectral range. 


\section{Introduction}

Kramers-Krönig (K-K) dispersion relations and sum rules [1, 2, 3, 4, have constituted for a long time fundamental tools in the investigation of light-matter interaction phenomena in condensed matter, gases, molecules, and liquids because they provide constraints able to check the self-consistency of experimental or model-generated data [5]. In particular these general properties allow to frame peculiar phenomena related to matter-matter or light-matter coupling that are very relevant at given frequencies, such as excitonic or polaritonic effects in solids [6], in the context of the interaction on the whole spectral range, showing that their dispersive and absorptive contributions are connected to all the other contributions in the rest of the spectrum[7]. Moreover by applying $\mathrm{K}-\mathrm{K}$ relations it is possible to invert optical data, i.e. acquiring knowledge on dispersive phenomena by measurements over all the spectrum of absorptive phenomena or viceversa [7, 9]. The conceptual foundations of such general properties, is the principle of causality[10] in the light-matter interaction, and the Titmarsch's theorem[1] provides the connection between the mathematical properties of the functions describing the physics in the time and frequency domains.

In spite of the ever increasing scientific and technological relevance of optical harmonic generation processes, and in general of nonlinear optical phenomena, relatively little attention has been paid to experimental investigation of the general properties of the corresponding nonlinear susceptibilities [12, 
13. 14, 15], because the research has usually focused on achieving high resolution in both experimental data and theoretical calculations. Rapidly developing technologies of tunable lasers allow to span larger and larger spectral ranges, so that the experimental investigations of frequency dependent nonlinear optical properties of matter is becoming more and more possible. In the context of this new class of experiments, K-K relations and sum rules could provide information on whether or not a coherent, common picture of the nonlinear properties of the material under investigation is available.

The first heuristic applications of K-K theory to nonlinear susceptibilities date back to the ' 60 [16, 17, 18, while a more systematic study has begun essentially in the last decade. Some authors have preferentially introduced the $\mathrm{K}-\mathrm{K}$ in the context of ab-initio or model calculations of materials properties [19, 20, 21, 22].

Other authors have used a more general approach able to give theoretical foundations of dispersion theory for nonlinear optics [23, 24, 25, 26, 27]. The instruments of complex analysis have allowed the definition of necessary and sufficient conditions for the applicability of K-K relations, which require the holomophicity of the susceptibility in the upper complex plane of the relevant frequency variable, given the form of the nonlinear susceptibility descriptive of the nonlinear phenomena under examination. It has also been shown that the asymptotic behavior of the nonlinear susceptibility, which can be obtained using the nonlinear Kubo-response function formalism [28], determines the number of independent pairs of K-K relations that hold simultaneously. 
In the cases $\mathrm{K}-\mathrm{K}$ do not hold, other techniques can be applied for the inversion of optical data [2, 29]. Combining K-K relations and knowledge of the asymptotic behavior of the nonlinear susceptibility sum rules for nonlinear optics, obtained by other authors by following different strategies [30], can be naturally derived along the same lines of the linear case.

Recent theoretical advances [31, 32] have generalized the results obtained for the second [27] and third harmonics [33] by showing arbitrary order harmonic generation susceptibilities $\chi^{(n)}(n \omega ; \omega, \cdots, \omega)$ are holomorphic in the upper complex $\omega$ plane, and asymptotically decrease as $\omega^{-2 n-2}$; therefore $\mathrm{K}-\mathrm{K}$ relations hold for all the moments $\omega^{2 \alpha} \chi^{(n)}(n \omega ; \omega, \ldots, \omega)$ (from now on instead of $\chi^{(n)}(n \omega ; \omega, \ldots, \omega)$ we use the simpler notation $\left.\chi^{(n)}(n \omega)\right)$ with $0 \leq \alpha \leq n$. From these a total of $2 n+2$ sum rules for the moments of the real and imaginary part of the susceptibilities can be derived, only one (the $(2 n+1)^{t h}$ of the imaginary part) being different from zero and descriptive of the inner structure of the material under investigation. The previous results have been recently extended [34] to the wider class of the moments of the $k^{\text {th }}$ powers of harmonic generation susceptibility, which can be written as $\omega^{2 \alpha}\left[\chi^{(n)}(n \omega)\right]^{k}$. In this case $\mathrm{K}-\mathrm{K}$ relations hold if $0 \leq \alpha \leq k(n+1)-1$, and therefore a total of $2 k(n+1)-2$ sum rules can be derived, with the $(2 k(n+1)-1)^{t h}$ moment of imaginary part giving the only nonzero summation over all the spectrum. The fundamental reason of this results is that if the harmonic generation susceptibility is holomophic in the upper complex $\omega$ plane, so are its powers. Higher powers of the susceptibility have faster 
asymptotic decrease, so that the limitations related to the finite range of real data are expected to be relaxed: this should be of particular benefit to the convergence of the sum rules, for which the consideration of the asymptotic behavior is more critical than for K-K relations [35, 7, 36].

The consideration of anchor points [37, 38] in the unknown part of $\chi^{(n)}(n \omega)$ 39] is a very promising technique for improving data inversion.

Until now, only in few investigations independent measurements of the real and imaginary parts of the harmonic generation susceptibilities have been performed for a relatively wide range 40, 41, 42, 43, and consequently the verification of the coherence of measured data by check of the self-consistency of $\mathrm{K}-\mathrm{K}$ relations is still of very limited use 42 .

In this paper we present the first analysis of harmonic generation data where the full potential of the generalized nonlinear K-K relations and sum rules for harmonic generation susceptibilities is exploited. We consider two published sets of wide spectral range experimental data of third harmonic generation susceptibility on different polymers, the polysilane [42] (frequency range: $0.4-2.5 \mathrm{eV}$ ) and the polythiophene [40] (frequency range: $0.5-2.0$ $e V)$.

In section 2 we apply K-K transformations to the real and imaginary part of the moments of the third harmonic generation susceptibility $\omega^{2 \alpha} \chi^{3}(3 \omega)$, with a suitable choices of $\alpha$, and describe the quality of the data inversion obtained in this way. We also perform the same analysis for $\omega^{2 \alpha}\left[\chi^{3}(3 \omega)\right]^{2}$, in order to prove for the first time on experimental data that $\mathrm{K}-\mathrm{K}$ relations 
hold also for the powers of the susceptibility, and show how many additional independent dispersion relations connect the real and the imaginary part. In section 3 we compute the sum rules for the experimental data, by calculating the integrals of the suitable moments of the real and imaginary parts of the susceptibility and of the $k^{t h}$ power of the susceptibilities, with $1 \leq k \leq 5$, and present a discussion on the issue of their convergence.

In section 4 we present our conclusions.

\section{Efficacy of generalized $K-K$ relations for data inversion}

In this study we base our calculations on two published sets of experimental data on third harmonic generation on polymers, where the real and imaginary part of the susceptibility were independently measured. The first data set refers to measurements taken on polisylane 42 and spans a frequency range of $0.4-2.5 \mathrm{eV}$. The second data set refers to measurements taken on polythiophene [40] and spans a frequency range of $0.5-2.0 \mathrm{eV}$.

In this paper we control the self-consistency of the two data sets by observing the efficacy of the K-K relations in inverting the optical data for the functions $\omega^{2 \alpha}\left[\chi^{3}(3 \omega ; \omega, \omega, \omega)\right]^{k}$, with $\mathrm{k}=1,2$. The theory 31, 32, 33, 34] prescribes convergence of the dispersion relations:

$$
\Re\left\{\left[\chi^{(n)}\left(n \omega^{\prime}\right)\right]^{k}\right\}=\frac{2}{\pi \omega^{\prime 2 \alpha}} \wp \int_{0}^{\infty} \frac{\omega^{2 \alpha+1} \Im\left\{\left[\chi^{(n)}(n \omega)\right]^{k}\right\}}{\omega^{2}-\omega^{\prime 2}} \mathrm{~d} \omega,
$$




$$
\Im\left\{\left[\chi^{(n)}\left(n \omega^{\prime}\right)\right]^{k}\right\}=-\frac{2}{\pi \omega^{\prime 2 \alpha-1}} \wp \int_{0}^{\infty} \frac{\omega^{2 \alpha} \Re\left\{\left[\chi^{(n)}(n \omega)\right]^{k}\right\}}{\omega^{2}-\omega^{\prime 2}} \mathrm{~d} \omega,
$$

only if $0 \leq \alpha \leq k(n+1)-1$, therefore in our case of $n=3 \mathrm{~K}-\mathrm{K}$ relations should work only if $0 \leq \alpha \leq 3$ if $k=1$ and $0 \leq \alpha \leq 7$ if we consider the second power of the susceptibility. In our study we do not assume any asymptotic behavior outside the data range, but use only the experimental data, because extrapolation is somewhat arbitrary and in K-K analysis can be quite problematic [8, 9]; we effectively apply truncated K-K relations and use a self-consistent procedure.

In the paper by Kishida et al. [42] a check of validity of the K-K relations was already performed by comparing measured and retrieved $\chi^{(3)}(3 \omega)$. Apart from the fact that our analysis considers also another data set, the consideration of the moments of the susceptibility is not a mere add-on to the work by Kishida et al. 42, but it represents a fundamental conceptual improvement: these additional independent relations are peculiar to the nonlinear phenomena, and provide independent double-checks of the experimental data that must be obeyed in addition to the conventional K-K relations.

We present in figures 1 and 2 the results of $\mathrm{K}-\mathrm{K}$ inversion for respectively the real and imaginary part of the third harmonic generation susceptibility data on polysilane. We observe that in both cases the retrieved data obtained with the choices $\alpha=0,1$ are almost indistinguishable from the experimental data, while for $\alpha=2$ and $\alpha=3$, the agreement is quite poor in the lower 
part of the spectrum: the error induced by the presence of the cut-off at the high frequency range becomes more critical in the data inversion for larger $\alpha$, since a slower asymptotic decrease is realized. We expect that inverting the data with the additional information given by anchor points located in the lower part of the data range these divergences can be cured. However, here our object is to deal with the worst case i.e. there is no a priori information about the phase of the complex nonlinear susceptibility at one or more fixed anchor points (fixed angular frequencies) 39]. From the theory we expect that for $\alpha=4$ no convergence should occur: actually we observe that, while the main features around $1.1 \mathrm{eV}$ are represented, there is no convergence at all for lower frequencies; the absence of a clear transition in retrieving performance between the $\alpha=3$ and the $\alpha=4$ case is due to the finiteness of the data range.

In figures 3 and 4 we show the comparison between retrieved and experimental data of third harmonic generation susceptibility on polythiophene for the real and imaginary part respectively. The dependence of the accuracy of quality of data inversion is similar to the previous case: for $\alpha=0,1$ the agreement is virtually perfect, while for $\alpha=2,3$ we have progressively worse performance in the low frequency range; anyway the peaks in the imaginary part are still well reproduced, while the dispersive structures in the real part are present but shifted towards lower values. In this case the quality of retrieved data for $\alpha=4$ is more distinct from what obtained with $\alpha=3$ than in the previous data set. The inversion with $\alpha=4$ presents a notable 
disagreement in the whole lower half of the data range for both real and imaginary part, in particular we see that in figure 3 the dispersive structure is absent, while the main peak in figure 4 is essentially missed.

Usually it is likely to expect that only the real or the imaginary part of the nonlinear susceptibility has been measured. Then normal procedure is to try data inversion using $\mathrm{K}-\mathrm{K}$ in order to calculate the missing part.

The results on figures $1-4$ confirm that best convergence is obtained when using conventional K-K, therefore these should generally be used to obtain a first best guess for the inversion of optical data, and should be used as seed for any self-consistent retrieval procedure; nevertheless if there is good agreement with the inversions obtained with higher values of $\alpha$, it is reasonable to conclude that the dispersion relations provide much more robust results. In this sense, the two data sets here analyzed are quite good in terms of self-consistency.

We underline that if on one side considering a higher power of the susceptibility implicitly filters out noise and errors in the tails of the data, on the other side experimental errors in the relevant features of the spectrum, peaks for the imaginary part and dispersive structures for the real part, are greatly enhanced if higher powers of the susceptibility are considered; in the latter case consistency between $\mathrm{K}-\mathrm{K}$ inversion of different moments is expected to be more problematic than in the $k=1$ case. Therefore improved convergence for more moments will occur for the powers of the susceptibility $k>1$ only if the data are basically good. 
In figures 5 and 6 we show the results of K-K inversion for respectively the real and imaginary part of the second power of the third harmonic generation susceptibility on polysilane. Up to our knowledge this is the first analysis of this kind on experimental data. We observe that for $\alpha=0,1,2$ the agreement between experimental and retrieved data is almost perfect, while it gets progressively worse for increasing $\alpha$. Nevertheless as long as $\alpha \leq 6$ the main features are well reproduced for both the real and imaginary part and the retrieved data match well if the photon energy is $\geq 1.0 \mathrm{eV}$. The theory predicts convergence for $\alpha=7$ and divergence for $\alpha=8$ : in our analysis we have divergence also in the former case, and it is reasonable to attribute this to the cut-off in the high frequency range of the data, because a very high moment as the $14^{\text {th }}$ requires a very well defined asymptotic behavior.

In figures 7 and 8 we repeat the same analysis for the second power of the susceptibility data taken on the polythiophene: also in this case the agreement is very good if $\alpha=0,1,2$, but the narrower frequency range does not allow the data inversion if the very high moments are considered. If we consider the real part -figure 7 for $\alpha=3,4 \mathrm{~K}-\mathrm{K}$ data inversion provides a good reproduction of the experimental data for photon energies $\leq 0.7 \mathrm{eV}$; for $\alpha \geq 5$ there is no convergence in the lower half of the spectral range. For the imaginary part -figure 8 we can repeat the same observations, except that for $\alpha=5$ there is still good reproduction of the main features of the curve.

We emphasize that if experimentally only one of the real or imaginary 
part of the harmonic generation susceptibility has been measured, there is no direct use of $\mathrm{K}-\mathrm{K}$ relations relative to higher powers of the susceptibility, since the multiplication mixes the real and the imaginary parts. Therefore in this case the K-K relations for $k>1$ can be used as tests of robustness of the results obtained with the dispersion relations applied to the conventional susceptibility.

\section{$3 \quad$ Verification of Sum Rules}

Sum rules for optical functions which obey K-K relations can be generally obtained by combining the dispersion relations with the knowledge of their asymptotic behavior, which can be obtained with a detailed analysis of the

physics of the system at microscopic level, by applying the superconvergence theorem [11, 3, 7] to the dispersion relations.

In the case of arbitrary order harmonic generation processes, we have that for large values of angular frequency $\chi^{(n)}(n \omega) \approx \psi \omega^{-2 n-2}$ 31, 32, where $\psi$ is a material-dependent constant. Therefore from the K-K relations (11) and (2) it is possible to derive the following sum rules [34:

$$
\begin{gathered}
\int_{0}^{\infty} \omega^{2 \alpha} \Re\left\{\left[\chi^{(n)}(n \omega)\right]^{k}\right\} \mathrm{d} \omega=0,0 \leq \alpha \leq k(n+1)-1 \\
\int_{0}^{\infty} \omega^{2 \alpha+1} \Im\left\{\left[\chi^{(n)}(n \omega)\right]^{k}\right\} \mathrm{d} \omega=0,0 \leq \alpha \leq k(n+1)-2
\end{gathered}
$$




$$
\int_{0}^{\infty} \omega^{2 k(n+1)-1} \Im\left\{\left[\chi^{(n)}(n \omega)\right]^{k}\right\} \mathrm{d} \omega=-\frac{\pi}{2} \psi^{k},
$$

The verification of linear sum rules [3, 4, 5, 7] from experimental data is usually hard to obtain because of the critical contributions given by the outof-range asymptotic part of the real or imaginary part of the susceptibility under examination [35, 36]; however in the case of linear optics information of the response of the material to very high frequency radiation can be obtained with synchrotron radiation [7. In general a good accuracy in the verification of sum rules is more difficult to achieve than for $\mathrm{K}-\mathrm{K}$ relations, therefore a positive outcome of this test provide a very strong argument to support the quality and the coherence of the experimental data.

In the case of harmonic nonlinear processes the technical limitations for achieving information for a very wide frequency range are very severe, and the verification of the sum rules is critical, especially for those involving relatively large values of $\alpha$ which determine a slower asymptotic decrease. Nevertheless, if we consider increasing values of $k$, the integrands in the equations (3), (4), and (15) have a much faster asymptotic decrease, so that the missing high-frequency tails tend to become negligible. Therefore we expect that for a given $\alpha$ the convergence of the sum rules should be more accurate for higher values of $k$, if we assume that the main features of the spectrum are well reproduced by the experimental data, as explained in the previous section. 
We first focus on the vanishing sum rules (3)-(44). In order to have a measure of how precisely the vanishing sum rules are obeyed for the two experimental data sets under examination, we introduce the dimensionless quantities $Z_{\Re}$ and $Z_{\Im}$ :

$$
\begin{gathered}
Z_{\Re}(\alpha, k)=\left|\frac{\int_{\omega_{\min }}^{\omega_{\max }} \omega^{2 \alpha} \Re\left\{\left[\chi^{(3)}(3 \omega)\right]^{k}\right\} \mathrm{d} \omega}{\int_{\omega_{\min }}^{\omega_{\max }} \omega^{2 \alpha}\left|\Re\left\{\left[\chi^{(3)}(3 \omega)\right]^{k}\right\}\right| \mathrm{d} \omega}\right|, \\
Z_{\Im}(\alpha, k)=\left|\frac{\int_{\omega_{\min }}^{\omega_{\max }} \omega^{2 \alpha+1} \Im\left\{\left[\chi^{(3)}(3 \omega)\right]^{k}\right\} \mathrm{d} \omega}{\int_{\omega_{\min }}^{\omega_{\max }} \omega^{2 \alpha+1}\left|\Im\left\{\left[\chi^{(3)}(3 \omega)\right]^{k}\right\}\right| \mathrm{d} \omega}\right| .
\end{gathered}
$$

Low values of $Z_{\Re}(\alpha, k)$ and $Z_{\Im}(\alpha, k)$ imply that the negative and positive contributions to the corresponding sum rule cancel out quite precisely compared to their total absolute value. The two data sets of the polymers have quite different performances in the verification of these sum rules.

We present in figures 9 and 10 the results obtained with the data taken of polysilane by considering $1 \leq k \leq 5$ for respectively the sum rules of the real and the imaginary part: we can draw very similar conclusions for both cases. We see that generally for a given $\alpha$, we have a better convergence when a higher $k$ is considered, with a remarkable increase in the accuracy of the sum rules for $k \geq 3$. Consistently with the argument that the speed of the asymptotic behavior is critical in determining the accuracy of the sum rule, we also have generally a decrease in the quality of the convergence to zero when, for a given $k$, one considers higher moments, thus increasing the value of $\alpha$. Particularly impressive is the increase of performance in the 
convergence of the sum rules of $\chi^{(3)}(3 \omega)$ for both the real $(2 \alpha=0,2,4,6)$ and the imaginary part $(2 \alpha+1=1,3,5)$ when we consider $k=4,5$ instead of $k=1$ : the values of $Z_{\Re}$ and $Z_{\Im}$ decrease of more than three orders of magnitude in all cases considered.

In figures 11 and 12 we present the corresponding results for the experimental data taken of polythiophene. Most of the sum rules computed with this data set show a very poor convergence to zero, because the correspond$\operatorname{ing} Z_{\Im}$ and $Z_{\Re}$ are above $10^{-1}$; nevertheless we can draw conclusions similar to the previous case in terms of change of the accuracy of the convergence for different values of $k$ and $\alpha$; consistently with the relevance of the asymptotic behavior, the precision increases for increasing $k$ and for decreasing $\alpha$. But in this case for a given $\alpha$ the improvement in the convergence of the sum rules obtained by considering a high value of $k$ instead of $k=1$ is generally small, being in most cases the decrease of $Z_{\Im}$ and $Z_{\Re}$ below or around an order of magnitude.

We observe that the bias between the performances of the two data sets in the verification of the vanishing sum rules is extremely large, not comparable to the discrepancies found in the analysis of the $\mathrm{K}-\mathrm{K}$ relations. We can guess that the worse performance of the data on polythiophene can be mainly attributed to their less complete representation of the relevant nonlinear electronic transitions of the material; as previously stated, the data extension is critical in the verification of sum rules. This result is consistent with the previously presented slightly worse performance of this data set in 
the K-K inversion of the second power of $\chi^{(3)}(3 \omega)$, where the relevance of the out-of-range data is also quite prominent.

Finally, from equation 5 it is possible to obtain a simple relation between the non vanishing sum rules for $\left[\chi^{(n)}(n \omega)\right]^{k}$ and the $k^{t h}$ power of the non vanishing sum rule for the conventional susceptibility $\chi^{(n)}(n \omega)$ :

$$
-\frac{2}{\pi} \int_{0}^{\infty} \omega^{2 k(n+1)-1} \Im\left\{\left[\chi^{(n)}(n \omega)\right]^{k}\right\} \mathrm{d} \omega=\left[-\frac{2}{\pi} \int_{0}^{\infty} \omega^{2 n+1} \Im\left\{\chi^{(n)}(n \omega)\right\} \mathrm{d} \omega\right]^{k}
$$

For both of the two data sets considered in our work these relations do not hold for $1 \leq k \leq 5$. The equation 8 relates the slowest converging sum rules for each $k$, therefore it is reasonable to explain the poor performances of the experimental data in reproducing this theoretical results with the finite range of data under examination.

\section{Conclusions}

In this study we have performed the first thorough analysis of generalized $\mathrm{K}-\mathrm{K}$ relations and sum rules on experimental data of third harmonic generation. We have tested the consistency between the theory and experimental data in the worst case, which however is usually most typical, namely data on limited spectral range without extrapolations beyond the measured range, and without any knowledge of anchor points [37, 38, 39]. We have considered two data sets of comparable spectral range referring to independent 
measurements of $\Re\left\{\chi^{(3)}(3 \omega)\right\}$ and $\Im\left\{\chi^{(3)}(3 \omega)\right\}$ performed on two polymers, the polysilane [42], whose data span a frequency range of $0.4-2.5 \mathrm{eV}$ and the polythiophene [40, whose data span a frequency range of $0.5-2.0 \mathrm{eV}$

We have inverted the optical data of the susceptibility using applying truncated $\mathrm{K}-\mathrm{K}$ relations with a self-consistent procedure and have verified for the first time that K-K relations hold consistently also for the moments of the susceptibility $\omega^{2 \alpha} \chi^{(3)}(3 \omega)$ with $0 \leq \alpha \leq 3$, as predicted by the general theory [31, 32, 33]; the two data sets show very similar performances. The precision of the data retrieved with $\mathrm{K}-\mathrm{K}$ relations is good in the upper part of the spectrum for all moments, while for $\alpha=2,3$ there is a disagreement with the experimental data in the very low portion of the spectrum, mainly due to the absence of data descriptive of the asymptotic behavior of the optical functions; nevertheless the main features of the data are well reproduced by the dispersion relations. The agreement between the results of the dispersion relations for the various moments of the susceptibility under examination provide fundamental information on the robustness of the retrieved data and on the self-consistency of the experimental data.

We have repeated the same analysis for $\omega^{2 \alpha}\left[\chi^{(3)}(3 \omega)\right]^{2}$, in order to give the first experimental confirmation of recent theoretical findings that predict that K-K relations hold for $\omega^{2 \alpha}\left[\chi^{(n)}(n \omega)\right]^{k}$ with $0 \leq \alpha \leq k(n+1)-1$, so in our case dispersions relations connect the real and imaginary part of $\omega^{2 \alpha}\left[\chi^{(3)}(3 \omega)\right]^{2}$ if $0 \leq \alpha \leq 7$. We have found that for the experimental third harmonic susceptibility measured on polysilane K-K relations hold with a high degree 
of precision for all the functions $\omega^{2 \alpha}\left[\chi^{(3)}(3 \omega)\right]^{2}$ with $0 \leq \alpha \leq 6$; the agreement between retrieved and experimental data is excellent for $0 \leq \alpha \leq 2$. In the case of polythiophene data, we can present similar conclusions, except that the agreement exists only for $0 \leq \alpha \leq 5$. The disagreement between the theory and the experimental fact can be safely attributed to the truncation occurring in the high frequency range, which mostly affects the convergence of the dispersion relations of the very high moments. K-K relations for higher powers of the susceptibility cannot be directly applied if measures on only one part of the susceptibility are available, but provide additional tests that inform on the completeness and self-consistency of measured and retrieved data.

We have investigated the validity of the sum rules that can be derived by combining the knowledge of the asymptotic behavior of $\chi^{(3)}(3 \omega)$ with the previously described sets of K-K relations for the moments of $\chi^{(3)}(3 \omega)$ and of its powers. The theory predicts that the integration in the semi-infinite positive $\omega$-domain of each even moments of the real part of the $k^{\text {th }}$ power of $\chi^{(n)}(n \omega)$ up to the $(2 k(n+1)-2)^{t h}$ gives 0 , and the same holds for the odd moments of the imaginary part of the $k^{\text {th }}$ power of the susceptibility up to the $(2 k(n+1)-3)^{t h}$; the only nonvanishing sum rule is the given by the integration of the $(2 k(n+1)-1)^{t h}$ moment of the imaginary part. In our case we have considered all the powers of $\chi^{(3)}(3 \omega)$ up to the fifth.

In order to evaluate the performance of the sum rules that the theory predicts to be vanishing, we have introduced an dimensionless measure of 
how precisely a sum rule computed on the experimental data converges to zero. Generally, for both the sum rules of the real and of the imaginary part, for a given $\alpha$, the convergence improves if we consider a higher value of $k$. Moreover, a decrease in the precision of the sum rules is realized if for a given $k$, higher moments are considered. The main reason for this behavior is in the concept that the faster the asymptotic decrease of the integrand of the sum rule under examination, the smaller the error due to the high-frequency cut-off. The two data sets for polymers differ greatly in the precision achieved in the verification of the sum rules, in many correspondent cases the polysilane data provide results that are better by orders of magnitude. The main reason for this discrepancy, much more relevant but coherent with the results obtained in the K-K study of $\left[\chi^{(3)}(3 \omega)\right]^{2}$, is the much stronger dependence of the sum rules precision on the position of high frequency range experimental cut-off relative to the saturation of the electronic transitions of the material: it is likely that the data on polythiophene are, apart from being narrower in absolute terms, less comprehensive relatively to the electronic properties of the material.

On the contrary, the experimental data on both polymers do not verify the non vanishing sum rules, which the theory predicts to give information about the structure of the material, because they do not obey the newly established consistency relation (8) which should hold for each value of the power $k$ considered for the susceptibility. The non vanishing sum rules involve the highest moments for which the integrations converge, therefore they are 
most strongly affected by the frequency range finiteness; improvements on the data range are then necessary to expect to obtain verification of these sum rules.

The constraints here analyzed are in principle universal, since they essentially derive from the principle of causality in the response of the matter to the external radiation, and so are expected to hold for any material; they provide fundamental tests of self-consistency that any experimental or model generated data have to obey; similar tests of coherence can be performed for other nonlinear optical processes, e.g. pump-and-probe[2, 13, 26, 44]. Verification of K-K relations and sum rules constitute unavoidable benchmarks for any investigation that addresses the nonlinear response of matter to radiation on a wide spectral range.

\section{Acknowledgments}

The authors would like to express their cordial thanks to Dr. Hideo Kishida (Department of Advanced Materials Science, University of Tokyo) and Dr. Takao Koda (Professor emeritus, University of Tokyo) for providing the measured optical data on polysilane. 


\section{References}

[1] L. D. Landau, E. M. Lifshitz, and P. Pitaevskii, Electrodynamics of Continuous Media (Pergamon, Oxford, 1984).

[2] K.-E. Peiponen, E. M. Vartiainen, and T. Asakura, Dispersion, Complex Analysis and Optical Spectroscopy (Springer, Heidelberg, 1999).

[3] M. Altarelli, D. L. Dexter, H. M. Nussenzveig, and D. Y. Smith, Phys. Rev. B 6, 4502 (1972)

[4] M. Altarelli and D. Y. Smith, Phys. Rev. B 9, 1290 (1974)

[5] J. Shiles, T. Sasaki, M. Inokuti, and D. Y. Smith, Phys. Rev. B 57, $1612(1980)$

[6] G. Grosso and G. Pastori Parravicini, Solid State Physics (Academic Press, San Diego, Calif., 2000)

[7] F. Bassani and M. Altarelli, in Handbook of synchrotron radiation, Vol. 1A p. 463, ed. E. E. Koch (North Holland, Amsterdam, 1983).

[8] K.-E. Peiponen and E. M. Vartiainen, Phys. Rev. B 44, 8301 (1991)

[9] D. E. Aspnes, in Handbook of Optical Constants of Solids, Vol. I p. 89. (Academic, New York, 1998)

[10] P. W. Milonni, J. Phys. B: At. Mol. Opt. Phys. 35, R31 (2002) 
[11] H. M. Nussenzveig, Causality and Dispersion Relations (Academic Press, New York, 1972)

[12] Y.H Lee, A. Chavez-Pirson, S.W. Koch, H.M. Gibbs, S.H. Park, J. Morhange, A. Jeffrey, N. Peyghambarian, L. Banyai, A.C. Gossard, and W. Wiegmann, Phys. Rev. Lett. 57, 2446 (1985)

[13] F. S. Cataliotti, C. Fort, T. W. Hänsch, M. Inguscio, and M. Prevedelli, Phys. Rev. A 56, 2221 (1997)

[14] U. Gubler, C. Bosshard, P. Gnter, M.Y. Balakina, J. Cornil, J.L. Brdas, R. Martin and F. Diederich, Proceedings of Conference on Lasers and Electro-Optics (CLEO 2000), San Francisco, California, USA, Technical Digest CMI1, 44 (2000).

[15] M. Martinelli, L. Gomes, and R. J. Horowicz, Appl. Opt. 39, 6193 (2000)

[16] P. J. Price, Phys. Rev. 130, 1792 (1963)

[17] M. Kogan, Sov. Phys. JETP 16, 217 (1963)

[18] W. J. Caspers, Phys. Rev. 133, 1249 (1964)

[19] E. Ghahramani, D.J. Moss, J.E. Sipe, Phys. Rev. B 43, 9700 (1991)

[20] M. Sheik-Bahae, D.J. Hagan and E.W. Van Stryland, Opt. and Quant. Electr. 24, 1 (1992) 
[21] L. Kador, Appl. Phys. Lett. 66, 2938 (1995)

[22] J. L. P. Hughes, Y. Wang, and J. E. Sipe, Phys. Rev. B 55, 13630 (1997)

[23] K.-E. Peiponen, Phys. Rev. B 35, 4116 (1987)

[24] K.-E. Peiponen, Phys. Rev. B 37, 6463 (1988)

[25] F. Bassani and S. Scandolo, Phys. Rev. B 44, 8446 (1991)

[26] S. Scandolo and F. Bassani, Phys. Rev. B 45, 13257 (1992).

[27] S. Scandolo and F. Bassani, Phys. Rev. B 51, 6925 (1995)

[28] R. Kubo, J. Phys. Soc. Japan 12, 570 (1957)

[29] K.-E. Peiponen and J. J. Saarinen, Phys. Rev. A 65, 063810 (2002)

[30] V. Chernyak and S. Mukamel, J. Chem. Phys. 103, 7640 (1995)

[31] F. Bassani and V. Lucarini, Il Nuovo Cimento D 20, 1117 (1998)

[32] F. Bassani and V. Lucarini, Eur. Phys. J. B 17, 567 (2000)

[33] N. P. Rapapa and S. Scandolo, J. Phys.: Condens. Matter 8, 6997 (1996)

[34] J. J. Saarinen, Eur. Phys. J. B 30, 551 (2002)

[35] U. Fano and J. W. Cooper, Rev. Mod. Phys. 40, 441 (1968) 
[36] P. Alippi, P. La Rocca, and G.B. Bachelet Phys. Rev. B 55, 13835 (1997)

[37] R. K. Ahrenkiel, J. Opt. Soc. Am. 61, 1651 (1971)

[38] K. F. Palmer, M.Z. Williams, and B.A. Budde, Appl. Opt. 37, 2660 (1998)

[39] V. Lucarini, J. J. Saarinen, K.-E. Peiponen, Opt. Commun. (in press)

[40] W. E. Torruellas, D. Neher, R. Zanoni, G. I. Stegeman, F. Kajzar, and M. Leclerc, Chem. Phys. Lett. 175, 11 (1990)

[41] D. Guo, S. Mazumdar, G. I. Stegeman, M. Cha, D. Neher, S. Aramaki, W. Torruellas, and R. Zanoni, Mater. Res. Soc. Symp. Proc. 247, 151 $(1992)$

[42] H. Kishida, T. Hasegawa, Y. Iwasa, T. Koda, and Y. Tokura, Phys. Rev. Lett. 70, 3724 (1993)

[43] H. Kishida, M. Ono, K. Miura, H. Okamoto, M. Izumi, T. Manako, M. Kawasaki, Y.Taguchi, Y. Tokura, T. Tohyama, K. Tsutsui, and S. Maekawa, Phys. Rev. Lett. 87, 177401-1 (2001)

[44] F. Bassani and V. Lucarini, Eur. Phys. J. B 12, 323 (1999) 


\section{Figure captions}

Fig. 1: Efficacy of K-K relations in retrieving $\Re\left\{\chi^{(3)}(3 \omega)\right\}$ on polysilane

Fig. 2: Efficacy of K-K relations in retrieving $\Im\left\{\chi^{(3)}(3 \omega)\right\}$ on polysilane

Fig. 3: Efficacy of K-K relations in retrieving $\Re\left\{\chi^{(3)}(3 \omega)\right\}$ on polythiophene

Fig. 4: Efficacy of K-K relations in retrieving $\Im\left\{\chi^{(3)}(3 \omega)\right\}$ on polythiophene

Fig. 5: Efficacy of K-K relations in retrieving $\Re\left[\left\{\chi^{(3)}(3 \omega)\right\}\right]^{2}$ on polysilane

Fig. 6: Efficacy of K-K relations in retrieving $\Im\left[\left\{\chi^{(3)}(3 \omega)\right\}\right]^{2}$ on polysilane

Fig. 7: Efficacy of K-K relations in retrieving $\Re\left[\left\{\chi^{(3)}(3 \omega)\right\}\right]^{2}$ on polythiophene

Fig. 8: Efficacy of K-K relations in retrieving $\Im\left[\left\{\chi^{(3)}(3 \omega)\right\}\right]^{2}$ on polythiophene

Fig. 9: Convergence to 0 of the vanishing sum rules $\omega^{2 \alpha} \Re\left[\left\{\chi^{(3)}(3 \omega)\right\}\right]^{k}$ with $1 \leq k \leq 5$; data on polysilane

Fig. 10: Convergence to 0 of the vanishing sum rules $\omega^{2 \alpha+1} \Im\left[\left\{\chi^{(3)}(3 \omega)\right\}\right]^{k}$ with $1 \leq k \leq 5$; data on polysilane

Fig. 11: Convergence to 0 of the vanishing sum rules $\omega^{2 \alpha} \Re\left[\left\{\chi^{(3)}(3 \omega)\right\}\right]^{k}$ with $1 \leq k \leq 5$; data on polythiophene

Fig. 12: Convergence to 0 of the vanishing sum rules $\omega^{2 \alpha+1} \Im\left[\left\{\chi^{(3)}(3 \omega)\right\}\right]^{k}$ with $1 \leq k \leq 5$; data on polythiophene 


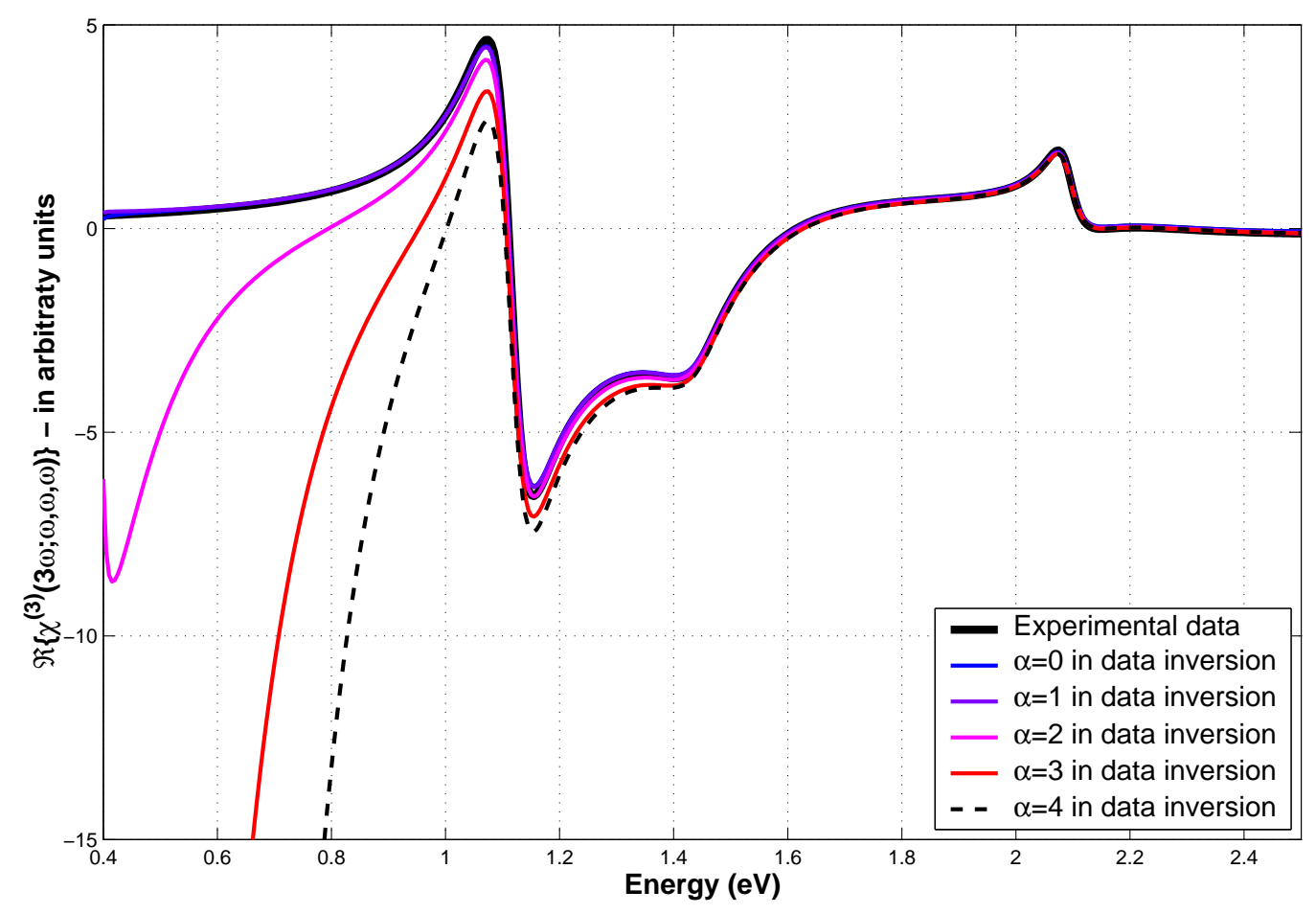

Figure 1: Lucarini, The Journal of Chemical Physics 


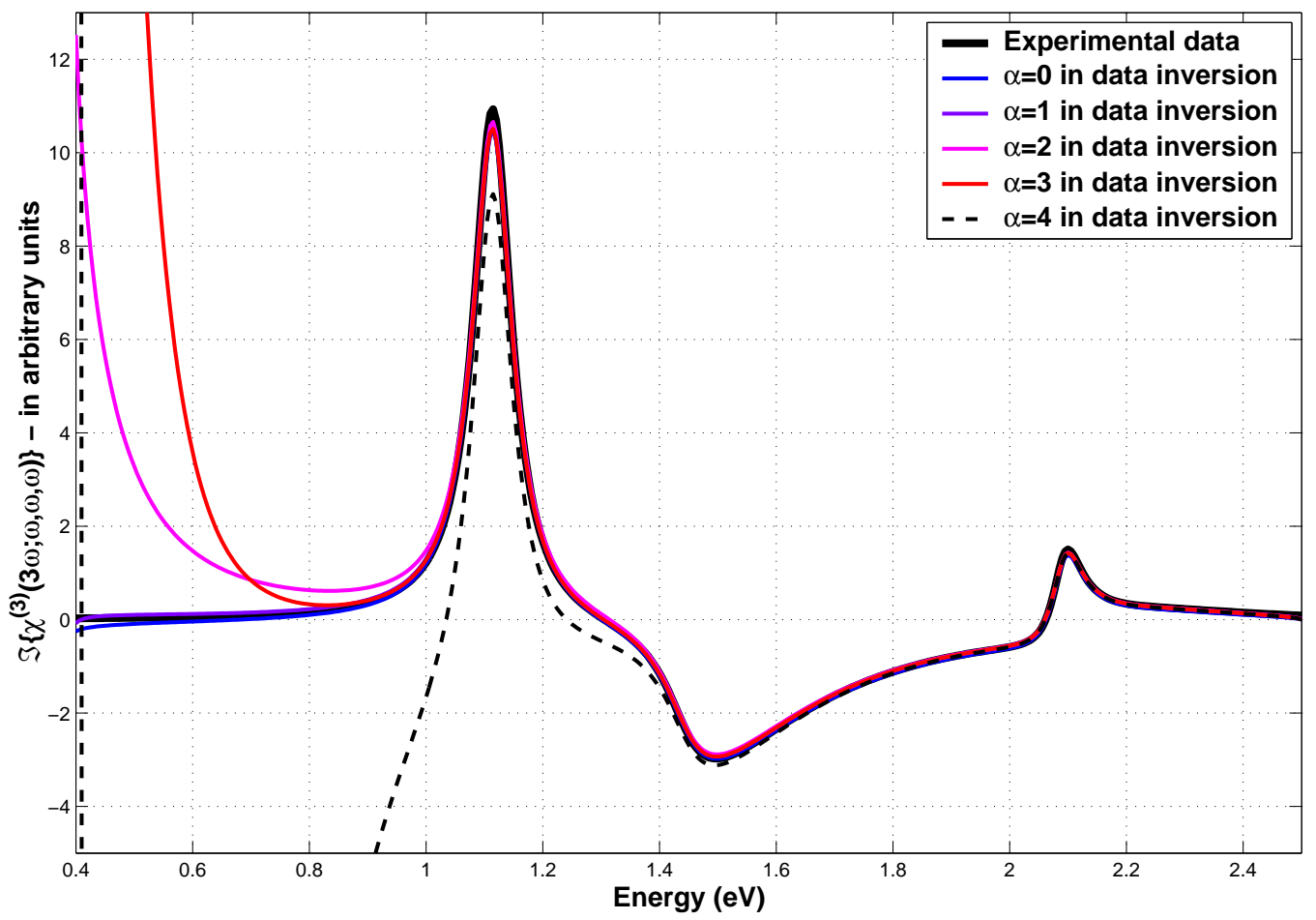

Figure 2: Lucarini, The Journal of Chemical Physics 


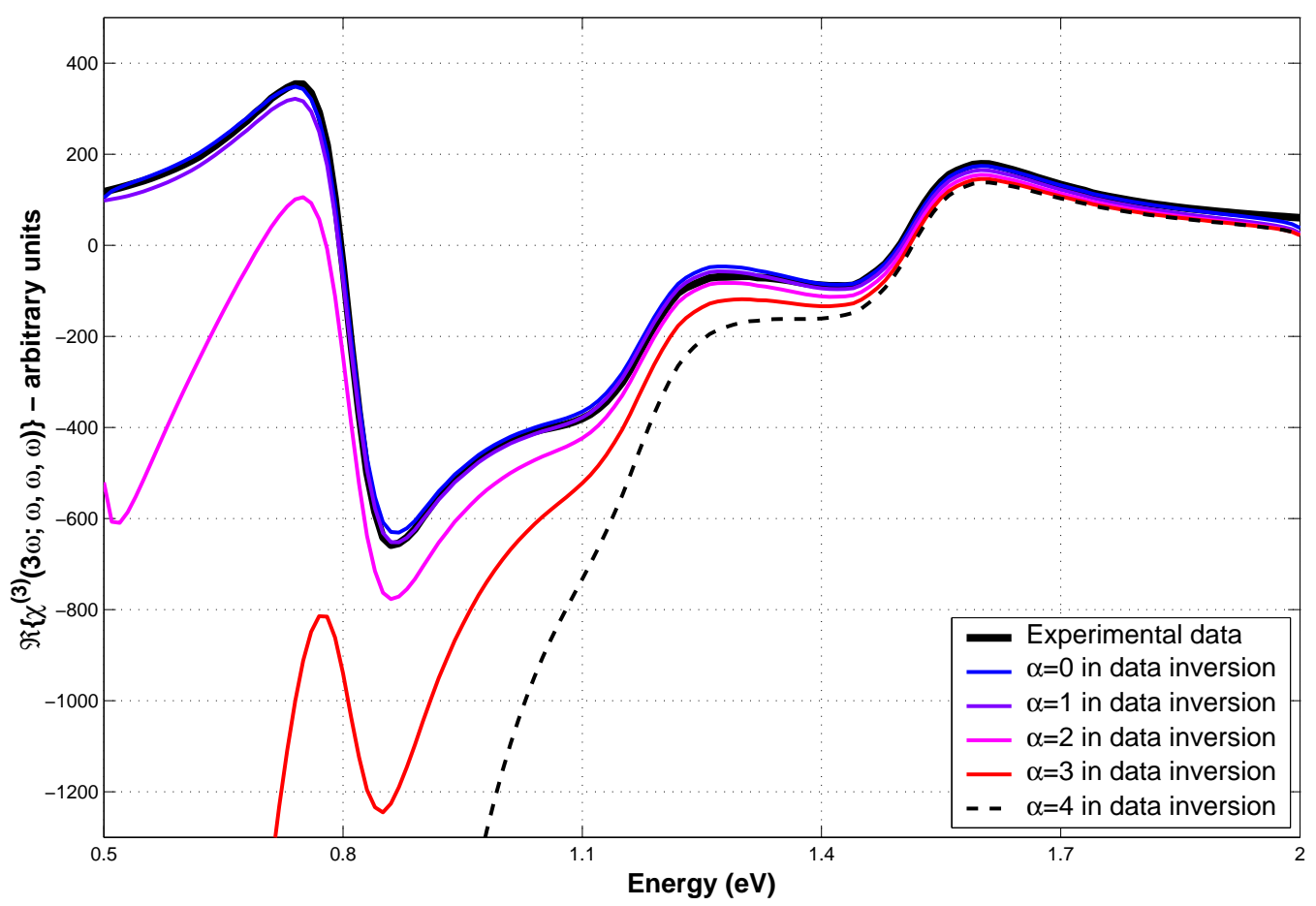

Figure 3: Lucarini, The Journal of Chemical Physics 


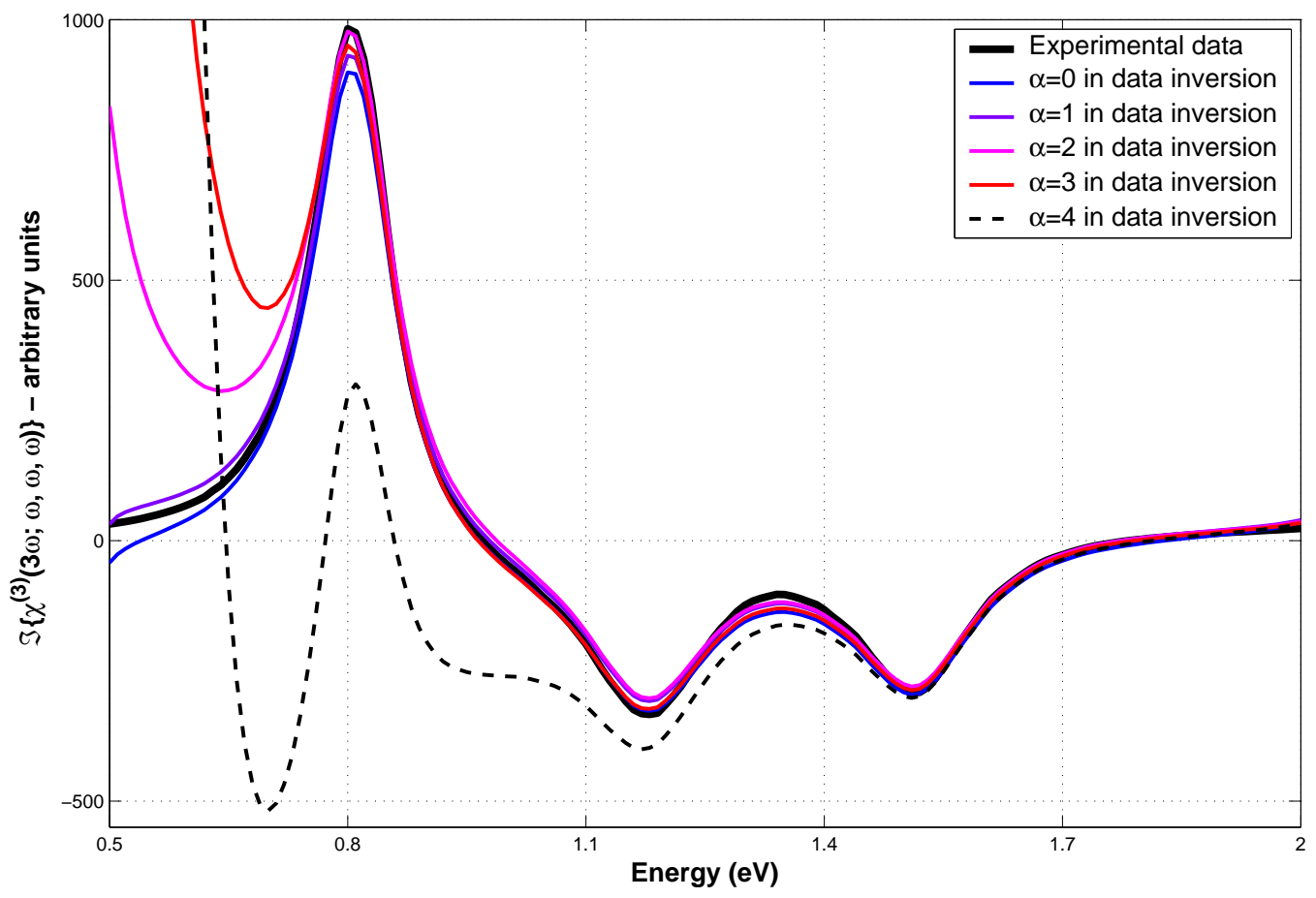

Figure 4: Lucarini, The Journal of Chemical Physics 


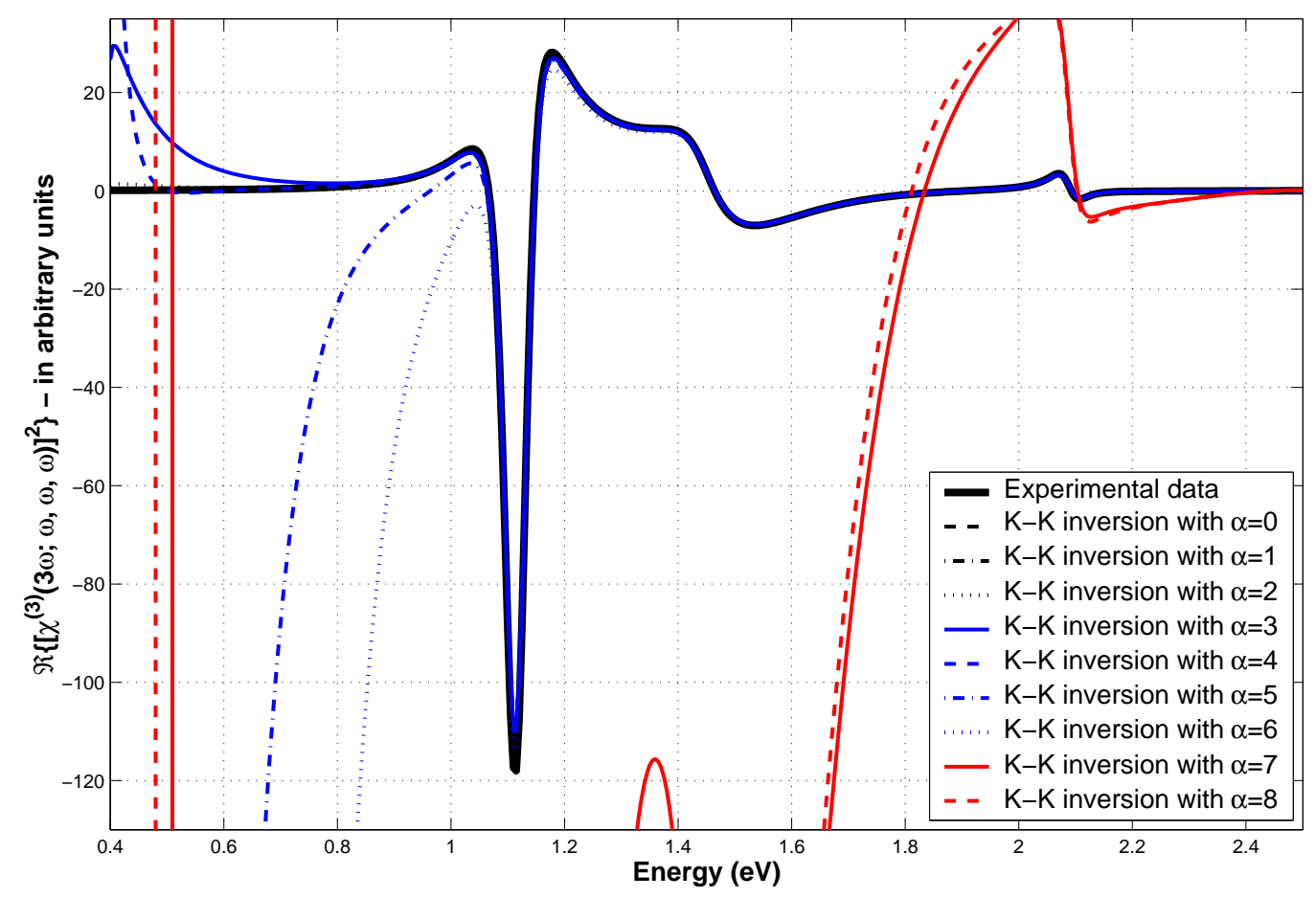

Figure 5: Lucarini, The Journal of Chemical Physics 


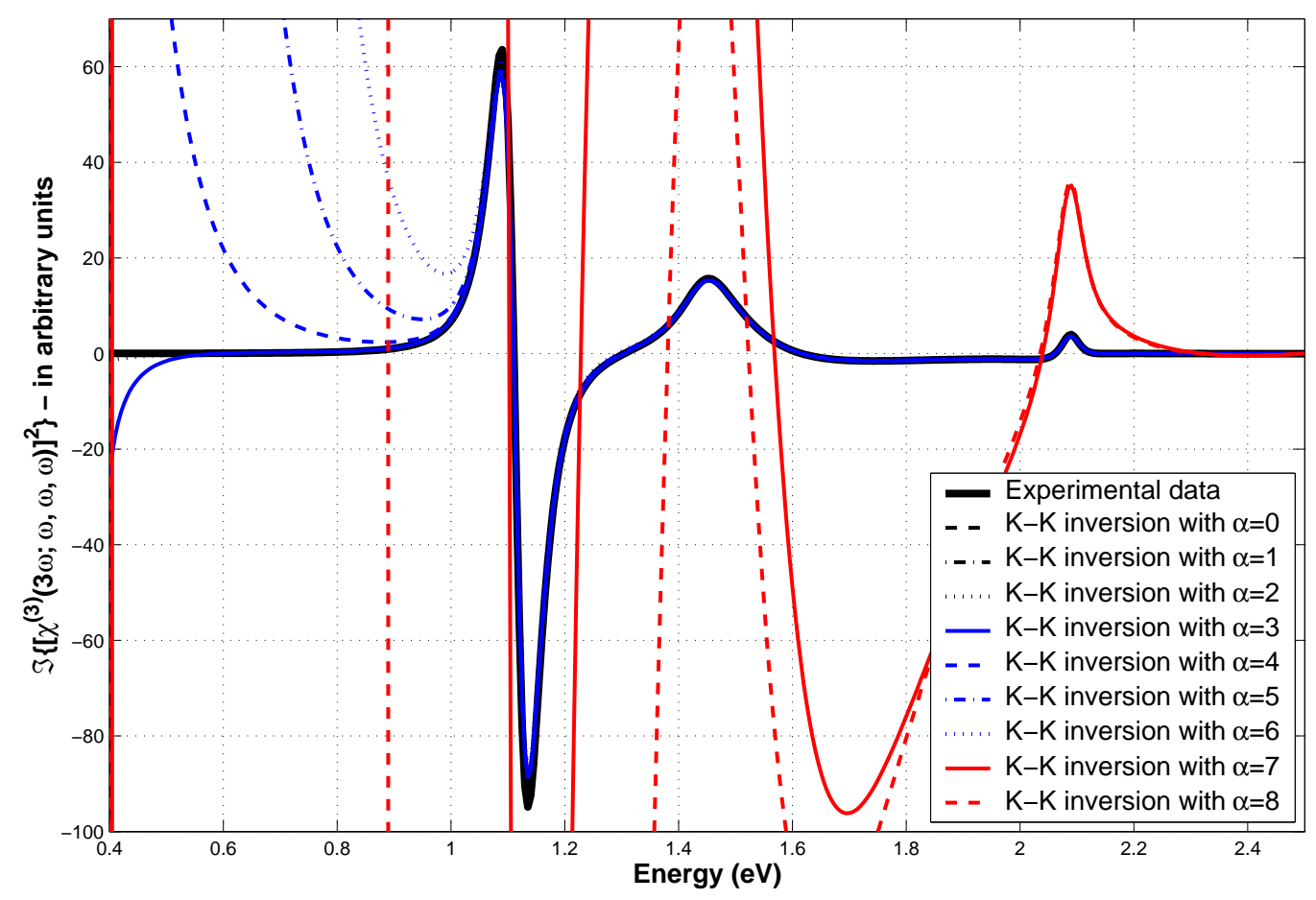

Figure 6: Lucarini, The Journal of Chemical Physics 


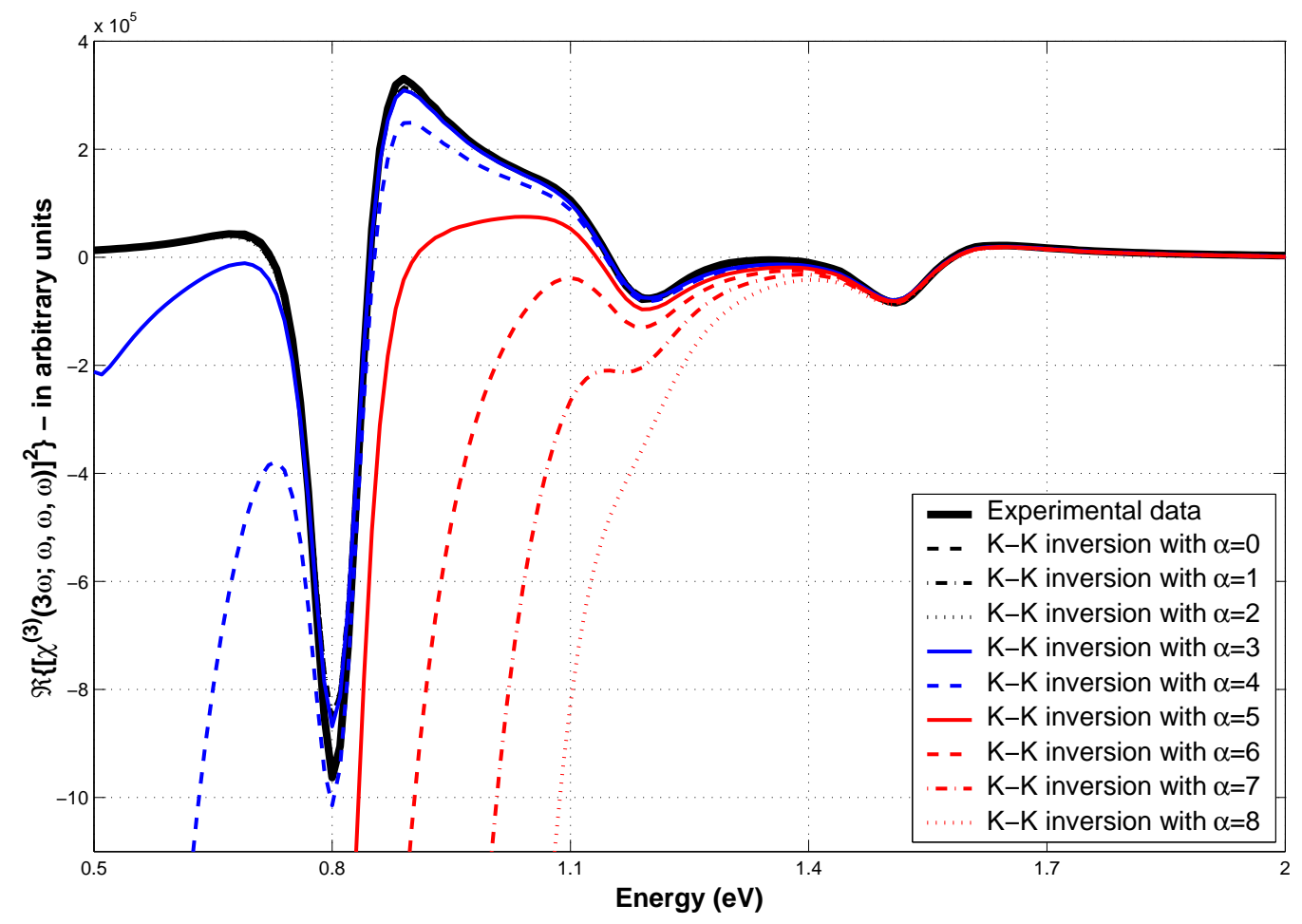

Figure 7: Lucarini, The Journal of Chemical Physics 


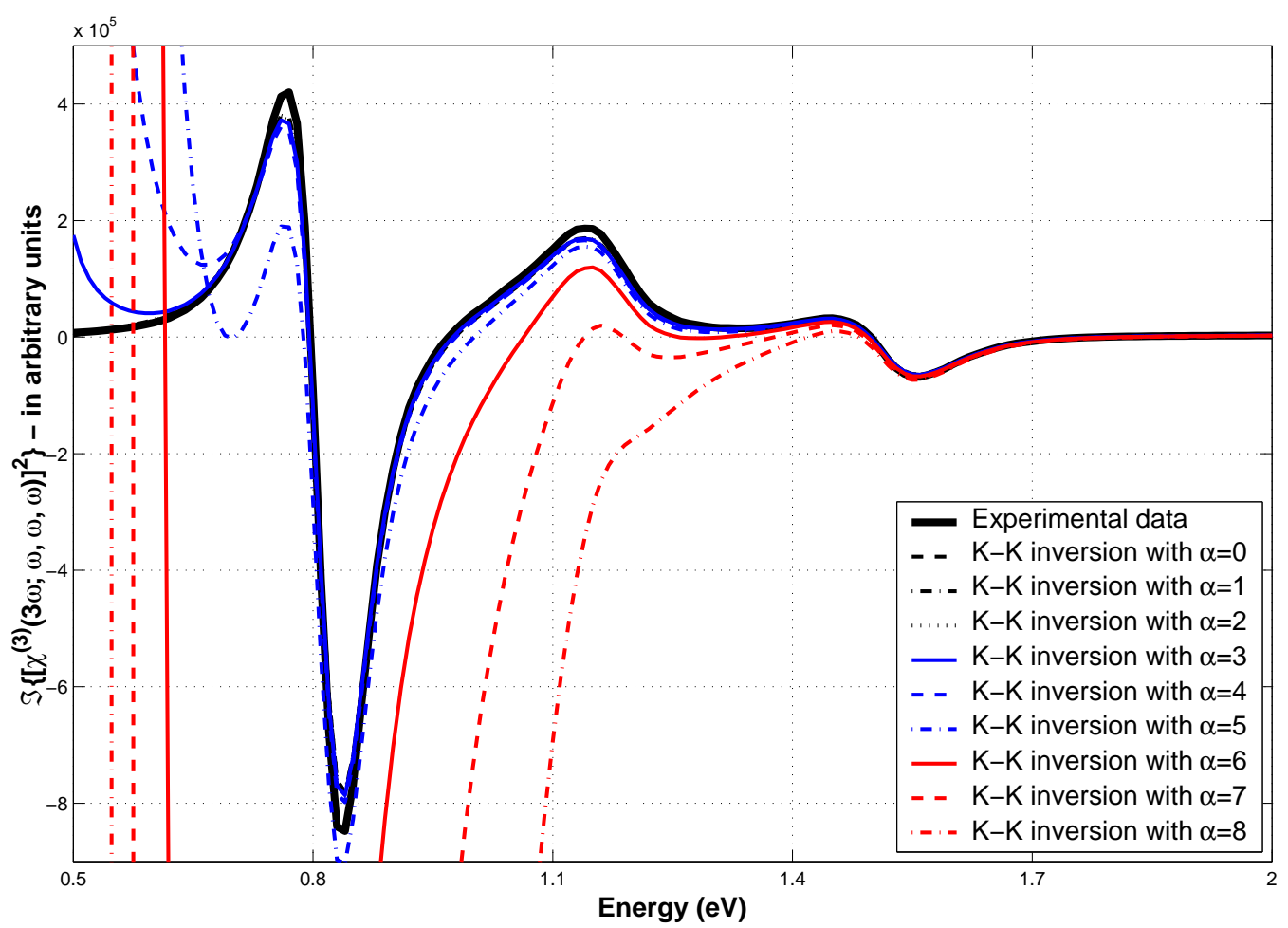

Figure 8: Lucarini, The Journal of Chemical Physics 


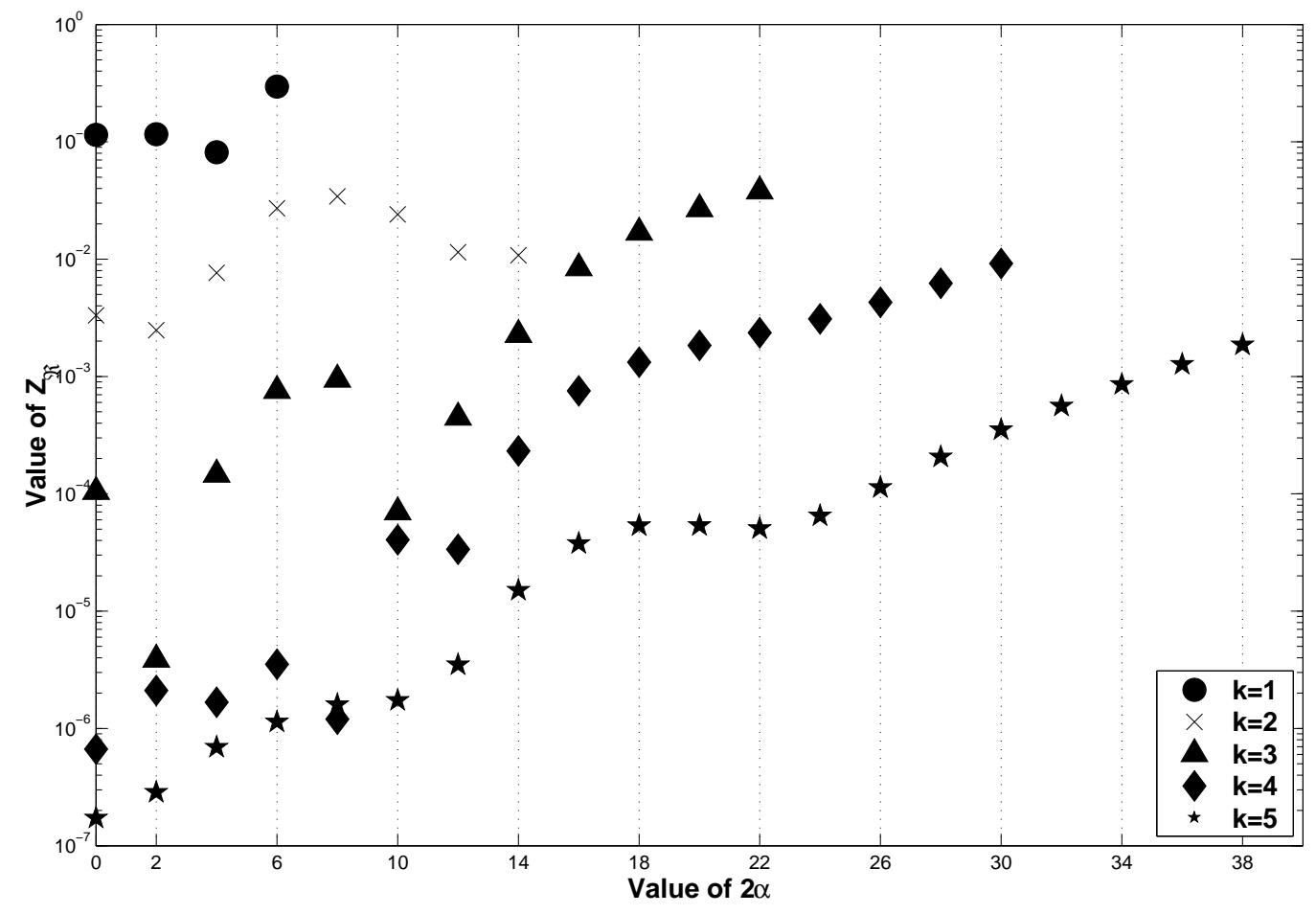

Figure 9: Lucarini, The Journal of Chemical Physics 


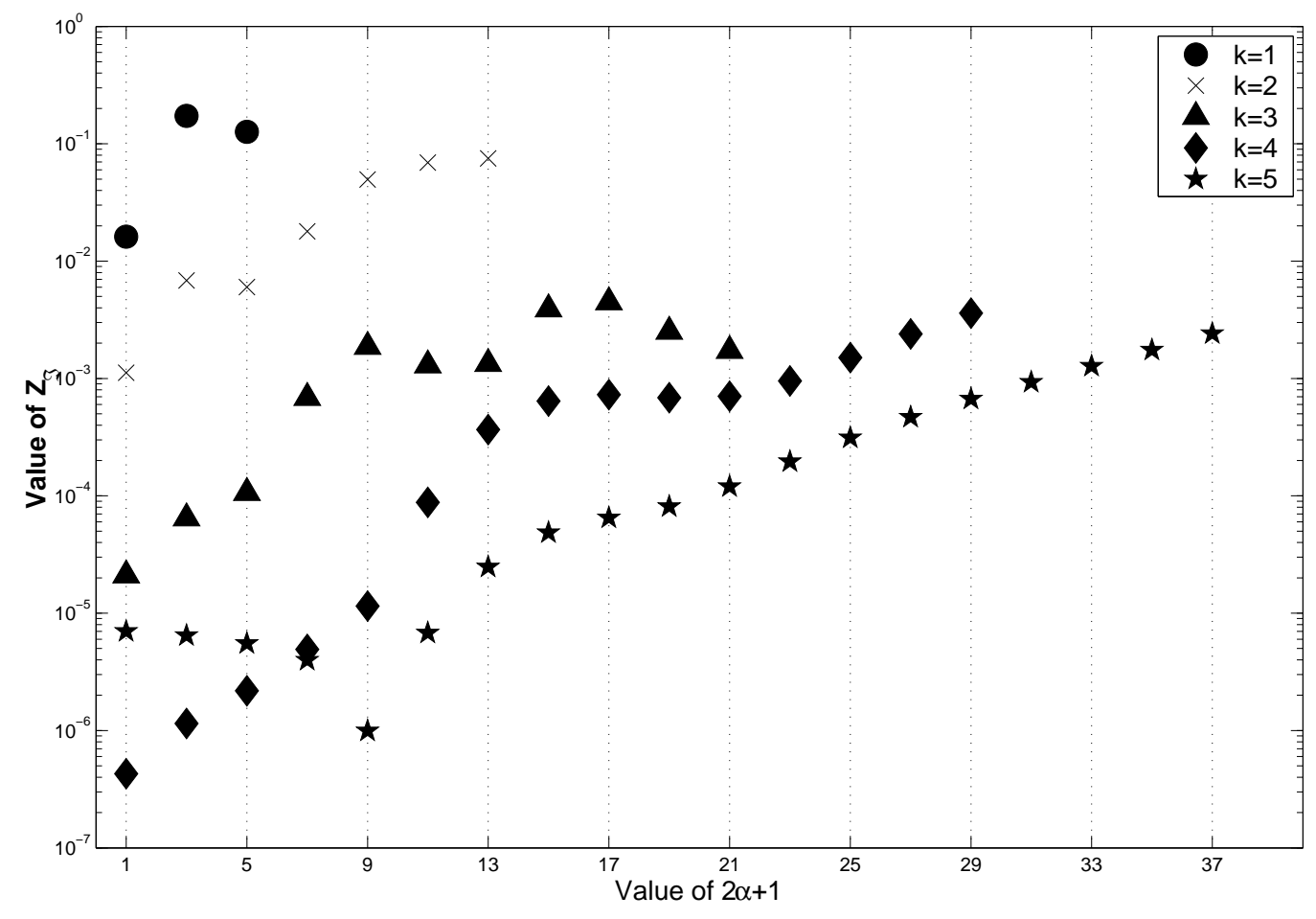

Figure 10: Lucarini, The Journal of Chemical Physics 


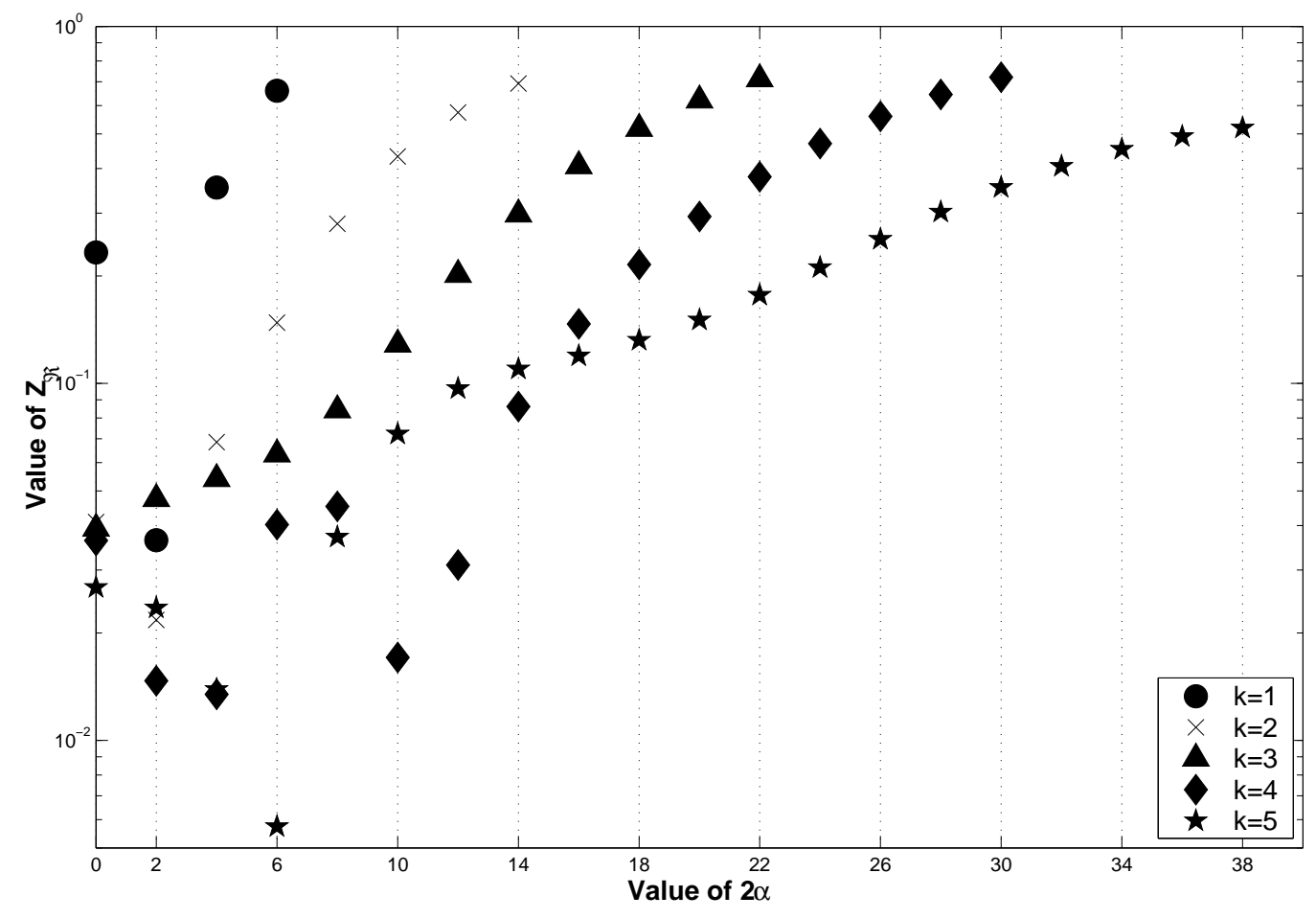

Figure 11: Lucarini, The Journal of Chemical Physics 


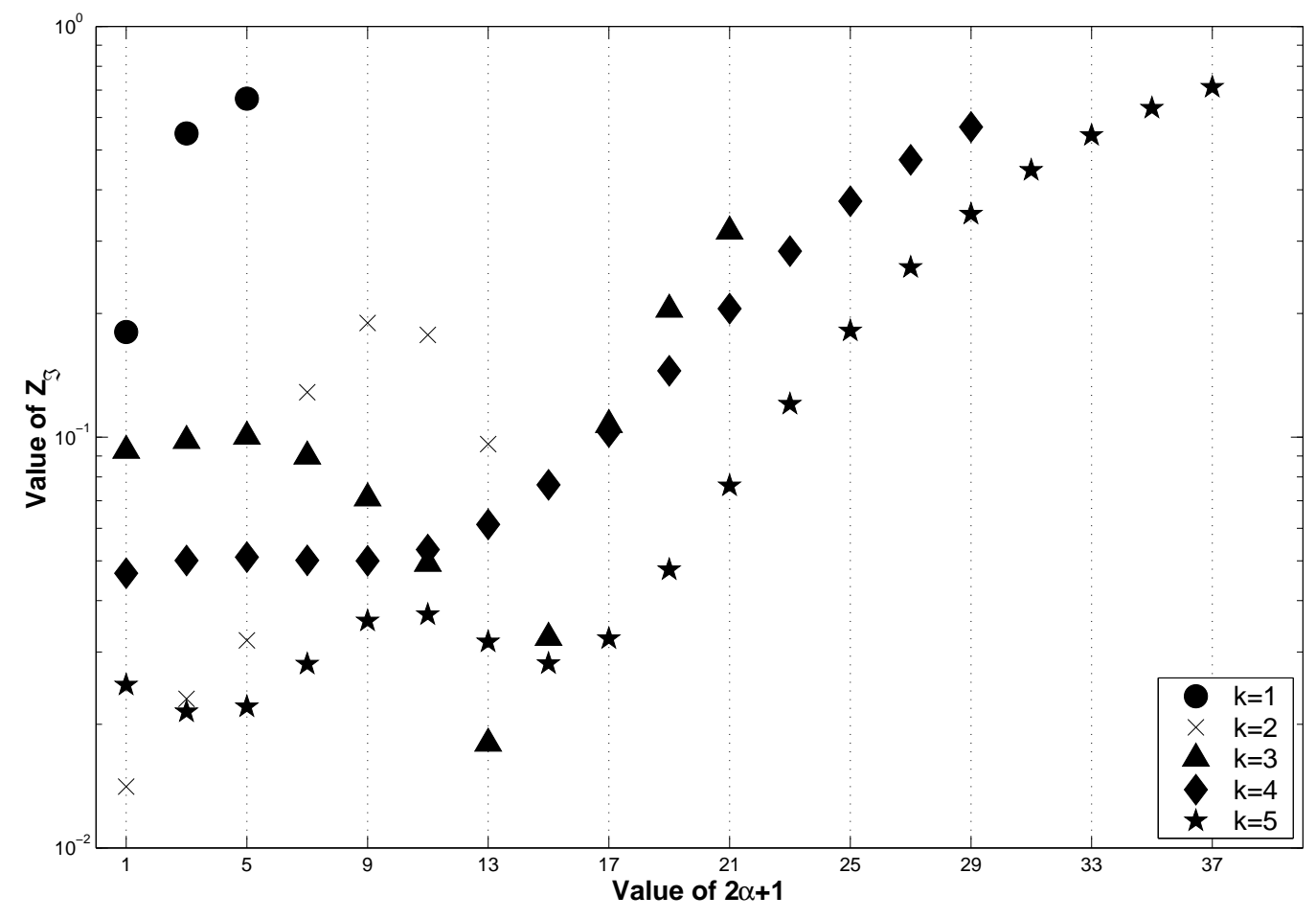

Figure 12: Lucarini, The Journal of Chemical Physics 